\title{
Evaluation of Ultrasound Accuracy in Axillary Lymph Node Involvement in Breast Cancer Patients
}

DOI: $10.21859 /$ mci-supp-119

\section{Keywords:}

Breast Cancer

Axillary Ultrasound

Sensitivity

Specificity

Positive Predictive Value

Negative Predictive Value
Asiie Olfatbakhsh ${ }^{1, *}$, Shahpar Haghighat ${ }^{1}$, Mansureh Khani ${ }^{1}$, Toktam Beheshtian ${ }^{1}$, Nasrin Alavi ${ }^{1}$, Fatemeh Sari ${ }^{1}$, Parisa Hosseinpour ${ }^{1}$

${ }^{1}$ Breast Diseases Department, Breast Cancer Research Center, Motamed Cancer Institute, ACECR, Tehran, Iran

${ }^{*}$ Corresponding author: Asiie Olfatbakhsh, Breast Diseases Department, Breast Cancer Research Center, Motamed Cancer Institute, ACECR, Tehran, Iran. E-mail: folfatbakhsh@yahoo.com

\begin{abstract}
Introduction: The status of the axillary lymph node is the most important factor in determining the prognosis of breast cancer. The aim of this study was to evaluate the sensitivity and specificity of Axillary Ultrasound (AUS) in the diagnosis of lymph node involvement in breast cancer patients referred to the Breast Cancer Research Center (BCRC) between March 2015 and Aug 2016.

Materials and Methods: Through a cross sectional study, all patients with a diagnosis of non-metastatic breast cancer with Stage I, II, IIIA and no chemotherapy before surgery were evaluated. The results of AUS and pathology after surgery were collected and analyzed using SPSS based on sensitivity and specificity formula.

Results: Of a total of 140 patients, the average age was 48.87 and the mean size of masses was $2.31 \mathrm{~cm}(0.4-10 \mathrm{~cm})$. Physical examination was positive in $14.6 \%$ of patients, positive AUS in $28.6 \%$ and pathologic axillary involvement in $39.2 \%$ of the patients. Sensitivity, specificity, PPV and NPV of AUS in diagnosing lymph node involvement were 55.5\%, 88.3 $\%, 75 \%$ and $76 \%$ respectively. Sensitivity, specificity, PPV and NPV of clinical examination were $31.48 \%, 97.67 \%, 89.4 \%$ and 69.4 . The combination of positive AUS and physical examination were along with pathologic axillary involvement in $92.3 \%$ of patients. False positivity and false negativity of AUS were $11.6 \%$ and $17.1 \%$ in all.

Conclusions: The sensitivity and specificity of AUS in our patients is the same as the results of other countries. Clinical examination alone has a high false negative and low sensitivity, however AUS in addition to physical examination has higher sensitivity which could predict axillary involvement and lead to axillary dissection without sentinel node biopsy. Negative AUS has about $24 \%$ false negativity and SLNB could not be omitted until the results of trials would be published.
\end{abstract}

